\title{
The use of optical coherence tomography angiography and optical coherence tomography to predict visual acuity in diabetic retinopathy
}

\author{
Joanna DaCosta ${ }^{1} \cdot$ Devangna Bhatia ${ }^{1} \cdot$ James Talks $^{1}$
}

Received: 20 October 2018 / Revised: 2 June 2019 / Accepted: 13 September 2019 / Published online: 7 October 2019

(c) The Author(s), under exclusive licence to The Royal College of Ophthalmologists 2019

\begin{abstract}
Introduction Diabetic macular ischaemia (DMI) is associated with reduced visual acuity. Limitations exist in assessing the ischaemic component of diabetic retinopathy. Optical coherence tomography angiography (OCTA) is a non-invasive imaging technique to distinguish retinal capillary layers and allow microvascular assessment. Evaluation of DMI is of prognostic significance when planning treatment regimens for diabetic maculopathy. The aims of this study were to evaluate OCTA to assess DMI and correlate findings with structural optical coherence tomography (OCT) features to predict visual acuity.

Methods Cross sectional study of fifty-seven eyes $(n=57)$ of thirty-seven subjects with diabetic retinopathy. $26 / 57$ (46\%) eyes had diabetic macular cysts. OCT and OCTA data from a single visit were analysed. Central retinal thickness, the presence of intraretinal cysts, ellipsoid zone (EZ) disruption, disorganisation of the retinal inner layers (DRIL), foveal avascular zone (FAZ) area, FAZ acircularity, and level of DMI in the superficial capillary plexus were graded according to ETDRS protocols. The deep vascular plexus was also graded for ischaemia. Correlations between measures and visual acuity were explored using regression models.

Results Median age was 55 years. Multiple regression analysis showed EZ disruption $(p<0.05)$, horizontal DRIL length $(p<0.01)$, DMI grade, and FAZ area $(p<0.1)$ correlated with visual acuity. Central retinal thickness and the presence of intraretinal cysts did not predict visual acuity in this study.

Discussion FAZ area, DMI grade, EZ disruption, and DRIL length correlate with visual acuity and could be incorporated into longitudinal clinical assessment of individual patients with diabetic retinopathy.
\end{abstract}

\section{Introduction}

Diabetic retinopathy (DR) is a leading cause of blindness amongst working aged adults [1]. Globally, it has been estimated that diabetic macular oedema (DMO) affects $\sim 23 \%$ of people with DR [2].

Diabetic macular ischaemia (DMI) is a feature of diabetic maculopathy, defined by enlargement of the foveal avascular zone (FAZ) and macular capillary non-perfusion and is associated with reduced visual acuity [3]. Evaluation of

$\triangle$ Joanna DaCosta

Joanna.DaCosta@nuth.nhs.uk

1 Newcastle Eye Centre, The Newcastle upon Tyne hospitals NHS Foundation Trust, Claremont Wing, Royal Victoria Infirmary, Queen Victoria Road, Newcastle upon Tyne NE1 4LP, UK
DMI maybe of prognostic significance when planning treatment regimens for DR and may also be helpful to explain a particular level of visual acuity. The ETDRS study established standards for assessment of DMI [4]. Fluorescein angiography has been conventionally used for the evaluation of DMI however fluorescein angiography assessment of the FAZ may not be reliable due to the requirement to obtain a sharply defined early photograph and is an invasive test.

Optical coherence tomography angiography (OCTA) is a non-invasive imaging technique, which can distinguish retinal capillary layers and allow microvascular assessment. Maybe by more accurately measuring the FAZ in both the superficial and deep venous plexus, which is not possible with FFA, a better understanding of how this relates to visual acuity can be gained. In addition, other OCT parameters may help with this assessment. 
Few studies have examined the relationship between visual acuity, cross sectional OCT, and OCTA derived metrics. The aims of this study were to evaluate OCTA to assess DMI and correlate findings with OCT findings to predict visual acuity.

\section{Methods}

This was a retrospective observational cohort study, reviewing patients seen at the Ophthalmology department in the Royal Victoria Infirmary, United Kingdom. The research and development office at the Newcastle upon Tyne Hospitals Foundation Health Trust approved this study and informed consent of patients was obtained. This study followed the principles of the Declaration of Helsinki.

This cross-sectional study involved analysing structural OCT and OCTA (Heidelberg Spectralis optical coherence tomography angiography, Heidelberg, Germany) data of the macular from a single visit of 57 eyes of 37 patients with different stages of DR. We excluded patients with concurrent retinal or macular conditions, uncontrolled glaucoma, and media opacities such as cataracts, corneal, and vitreous opacities. Patients were included who had a refractive error of between +2.0 and -2.0 dioptres spherical equivalent. The superficial vascular plexus (SVP) and deep vascular plexus (DVP) were segmented using the inbuilt software. The segmentation lines were checked for accuracy and adjusted manually if needed to account for attenuation of signal intensity associated with cystic spaces. Central retinal thickness (CRT) was defined as the average thickness $(\mu \mathrm{m})$ in the central $1 \mathrm{~mm}$ circle defined by ETDRS.

For the analysis of the cross-sectional OCT the following definitions were used. DMO was defined as a CRT > 300 $\mu \mathrm{m}$. The presence of intraretinal cysts was defined as the presence of localised hypo reflective areas. Ellipsoid zone (EZ) disruption was defined as horizontal length of disruptions involving the external limiting membrane and EZ (previously termed inner segment/outer segment photoreceptor junction). Disorganisation of the inner retinal layers (DRIL) was defined as inability to delineate the inner retinal layer boundaries within the central $1500 \mu \mathrm{m}$ region. FAZ area and perimeter was measured by exporting images and utilising image $\mathrm{J}$ software (http://imagej.nih.gov/ij) available in the public domain (National Institute of Health, USA). Acircularity index was defined as the ratio of FAZ perimeter to perimeter of a perfect circle with equal area. The level of ischaemia in the superficial capillary plexus was graded according to ETDRS protocols. The DVP was also graded for ischaemia. Correlations between these measures and visual acuity were explored with regression models. Statistical analysis was performed with Minitab ${ }^{\circledR}$ Statistical software. A sample size of forty or above was planned as determined by the software regression model to find a statistically significant result.

\section{Results}

The baseline characteristics of the 57 eyes of 37 patients are presented in Table 1 . The majority of the eyes did not have macular oedema $(72 \%)$, with the mean CRT being 282.5 $\mu \mathrm{m}$. This was to reduce the potentially difficulties of segmentation and assessment of OCT features when there is macular oedema. Table 2 shows a multivariable regression analysis of the correlation between $\log$ MAR visual acuity and OCT and OCTA derived parameters. Horizontal DRIL length $(p=0.01)$ and EZ disruption length $(p=0.04)$ were associated with worse visual acuity. The correlation of deep FAZ and EDTRS DMI is shown in Fig. 1. The FAZ area had a weak association $(p=0.08)$, with visual acuity (Fig. 2) and whilst regression analysis does show an association there is a large spread of results (Fig. 3).

With regard to co-relation between OCTA and OCT parameters multivariable regression analysis demonstrated FAZ area was associated with horizontal DRIL length and CRT $(p<0.001)$, not EZ disruption or the presence of IR cysts. Acircularity index was associated with intraretinal cysts $(p=0.006)$, not horizontal DRIL length, horizontal EZ disruption or CRT.

Table 1 Characteristics of the study population

\begin{tabular}{ll}
\hline Variables & $\begin{array}{l}\text { Total }(n=57) \text { eyes of } 37 \\
\text { patients }\end{array}$ \\
\hline Gender (\%): male/female & $15(41 \%) / 22(59 \%)$ \\
Age (years, median) & 55 \\
Lens status (\%): phakic/ & $43(75 \%) / 14(25 \%)$ \\
pseudophakic & \\
Diabetic retinopathy grade & \\
None & $3 / 57(5 \%)$ \\
Mild NPDR & $28 / 57(49 \%)$ \\
Moderate NPDR & $8 / 57(14 \%)$ \\
Severe NPDR & $5 / 57(9 \%)$ \\
PDR & $13 / 57(23 \%)$ \\
Macular cysts (\%) & $26 / 57(46 \%)$ \\
Diabetic macular oedema & $16 / 57(28 \%)$ \\
Central retinal thickness & $282.5 \mu \mathrm{m}$, range $196-429 \mu \mathrm{m}$ \\
(mean, range) & \\
CRT (mean, cysts present) & $282.7 \mu \mathrm{m}$ \\
CRT (mean, no cysts present) & $278.6 \mu \mathrm{m}$ \\
Prior treatment & \\
(Pan retinal photocoagulation, & $25 / 37(68 \%)$ \\
macular laser, intravitreal steroid & \\
or antiVEGF treatment) & \\
\hline
\end{tabular}


Table 2 Multivariable regression analysis of the correlation between $\log$ MAR visual acuity and OCT and OCTA derived parameters

\begin{tabular}{lc}
\hline Parameter & $p$-value \\
\hline FAZ area $\left(\mathrm{mm}^{2}\right)$ & 0.08 \\
Age & 0.11 \\
Central retinal thickness & 0.74 \\
Intraretinal cysts & 0.34 \\
Acircularity index & 0.97 \\
Horizontal DRIL length & 0.01 \\
Horizontal EZ disruption & 0.04 \\
ETDRS DMI grade & \\
Questionable & 0.07 \\
Mild & 0.09 \\
Moderate & 0.02 \\
Severe & 0.01 \\
\hline
\end{tabular}

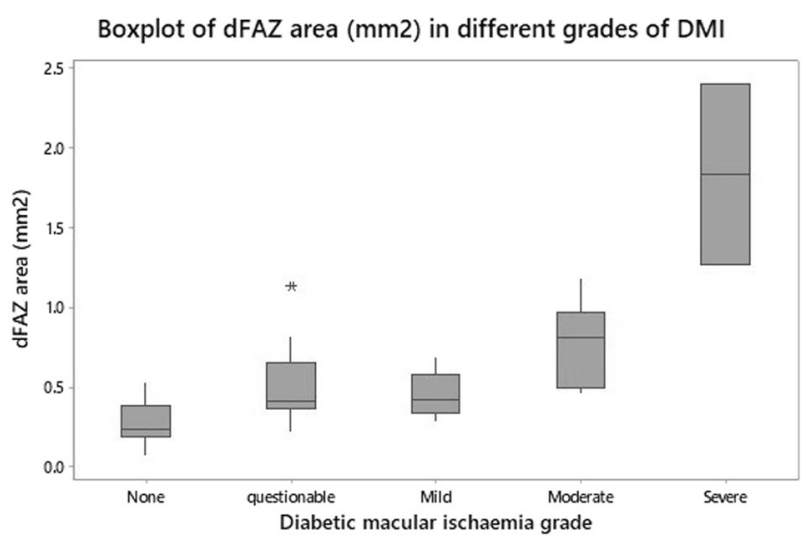

Fig. 1 Box plots of FAZ area $\left(\mathrm{mm}^{2}\right)$ in different ETDRS grades of DMI: none, questionable, mild, moderate, and severe. The bottom of the box represents the 25th percentile, the top of the box represents the 75th percentile, and the line in the middle of the box represents the 50th percentile. The whiskers represent the highest and lowest values that are not outliers or extreme values. The asterisk beyond the whiskers represents an outlier or extreme value

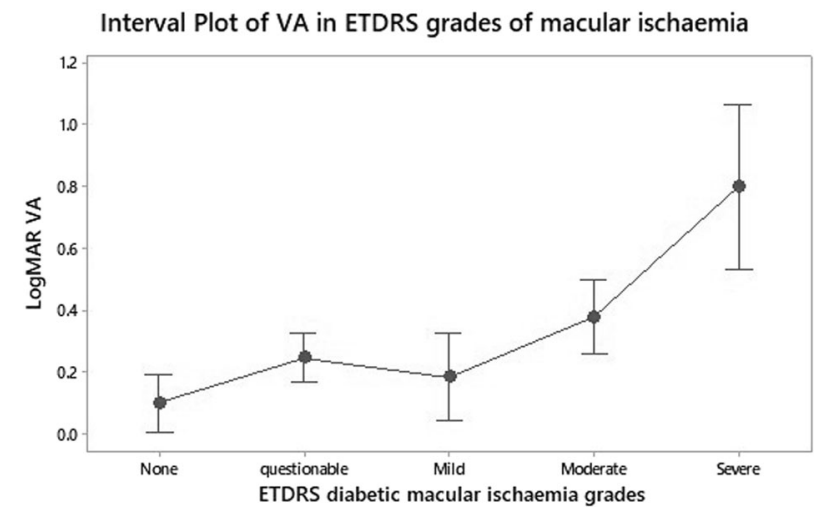

Fig. 2 Interval plot to assess and compare confidence intervals for logMAR visual acuity measured in different ETDRS diabetic macular ischaemic grades. A $95 \%$ confidence interval is shown for the mean of each group

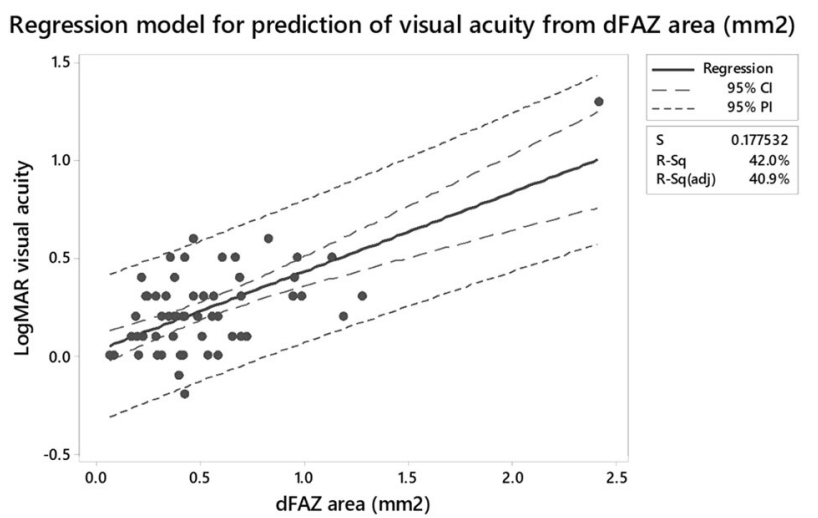

Fig. 3 Regression model for prediction of visual acuity from dFAZ area $\left(\mathrm{mm}^{2}\right)$. On the fitted line plot, the 95\% confidence (CI) and 95\% prediction intervals (PI) are displayed as dashed lines identifying the upper and lower limits of the intervals. The confidence interval represents a range of likely values for the mean response. The prediction interval represents a range of value for a single new observation and is wider as predicting a single response value is less certain. $S$ represents the standard error of the regression, the average distance that the observed values fall from the regression line

\section{Discussion}

Our study found an association with visual acuity and EZ disruption $(p<0.05)$, horizontal DRIL length $(p<0.01)$, DMI grade, and FAZ area $(p<0.1)$. FAZ area correlated better with ETDRS grades of either no ischaemia or severe ischaemia. By measuring a FAZ area associated with questionable, mild or moderate ETDRS ischaemia it was more challenging to predict visual acuity.

OCTA has enabled visualisation of the deep capillary plexus, previously undetectable with FFA. The association between retinal capillary loss and visual acuity is largely unknown, particularly with regard to confounding factors such as media opacity, macular oedema, and retinal remodelling. The FAZ is a specialised region of the retina containing the highest density of cone photoreceptors and high oxygen consumption [5]. In health, the size of the FAZ does not appear to determine visual function [6]. A large variability in FAZ size amongst individuals has also been reported [7]. An enlargement in FAZ size has been noted with age in healthy eyes and this does not appear to affect visual acuity [8]. FAZ area also varies with gender $[9,10]$. FAZ area on its own is therefore not likely to be a reliable marker to predict vision in DR although there is a trend with poorer vision and increasing FAZ size. Our results demonstrate a weak association between visual acuity and FAZ size in diabetics $(p<0.1)$.

Bresnick et al. quantified FAZ enlargement in diabetic patients utilising FFA and associated this with the severity of DR [11]. Sim et al. examined capillary non-perfusion by fluorescein angiography and found an association between reduced vision in only $15 \%$ of eyes with moderate to severe 
ETDRS-DMI grades of ischaemia [3]. Vision was preserved in eyes with milder degrees of ischaemia. Samara et al. conducted a retrospective observational case series demonstrating a correlation between visual acuity and FAZ area in both SVP and DVP [12]. They utilised automated algorithms utilising AngioAnalytic software so limiting direct comparison with our study.

FAZ area as determined by OCTA was significantly correlated with visual acuity in DR and retinal vein occlusion $(p<0.003)$ in a cross-sectional study [9]. The association was found to be modulated by age $(p<0.026)$ such that for a constant FAZ area a relatively older group of patients will have poorer vision. Multiple regression analysis with visual acuity as the outcome measure demonstrated that in addition to FAZ area and age, EZ disruption was also significantly associated with vision [9]. Lens status (phakic or pseudophakic), DRIL length, and the occurrence of IR cysts were not found to be significant predictors of vision with this model [9]. FAZ measurements were obtained using the entire volume scan but stratification of capillary networks into superficial and deep layers was not performed limiting comparison to our results [9]. However, our results agree with the findings of EZ disruption being associated with visual acuity and we also did not find an association with the occurrence of IR cysts and visual acuity. Our results differ, in that we found an association between DRIL length and visual acuity.

Image analysis methods and metrics such as FAZ circularity, vessel density (VD), and fractal dimension have been utilised to quantify retinal capillary networks from OCTA to assess abnormalities in diabetics. Acircularity index is defined as the ratio of FAZ perimeter to the perimeter of a circle with equal area [13]. A FAZ with perfect circularity has an acircularity index equal to 1 . In our study, we found no correlation between the acircularity index and visual acuity. This result agrees with Krawitz et al. who found a relationship between worsening stages of DR and higher acircularity index but no association with visual acuity in a retrospective study [14].

We did not examine macular VD as a predictor of visual acuity. We used an OCTA spectralis platform (Heidelberg Engineering) in this study. There appears to be no clear consensus on methodology for obtaining measurements using this device of vascular density that can be validated so limiting direct comparison with other studies. Various measures of VD have been previously reported utilising different OCTA devices and processing algorithms. In particular, automated VD using AngioAnalytic software has been reported [15]. To our knowledge, the exact algorithms the software utilises to generate measures has not been described. Dupas et al. studied the association between macular VD, as measured by OCTA, and visual acuity in a specific group of young type 1 diabetics ( $<40$ years) without macular oedema, who were poorly controlled and had severe progressive DR requiring PRP [15]. A weak correlation was observed between FAZ area and visual acuity. Their study utilised default automatic segmentation AngioVue OCTA system and AngioAnalytic software. They observed visual acuity depended mainly on VD in the DCP. Their study was limited in that a small selective sample of diabetics were included and the combined effects of past antiVEGF therapy and PRP may have confounded results [15].

Intercapillary area (ICA) is measured by the average of multiple contiguous areas without detectable vessels in the perifoveal region [16, 17]. Tang et al. conducted a crosssectional study to study the association between ICA and visual acuity [18]. Their findings suggest increased ICA measured from OCTA was associated with poorer visual acuity. The association with visual acuity was found to be stronger than that of FAZ area and VD and comparable with FAZ circularity. The relationship between ICA and visual acuity was unclear due to the cross-sectional design of the study and limitations were that cataract severity was not included [18]. In addition, images obtained from the deep capillary plexus were not included due to shadow projection artefacts. Dupas et al. hypothesised that there may be a threshold of macular non-perfusion in the DCP beyond which vision may be compromised [15]. This threshold may vary amongst individuals and be influenced by pathophysiological processes in a cumulative effect. In DR, the retinal microenvironment may be compromised due to cardiovascular and renal disease and interaction with individual genetic and environmental factors such as smoking resulting in a complex pathophysiological picture.

Visual acuity, traditionally tested with high contrast may not represent the optimal measure of visual function. Visual acuity loss may represent a late stage of functional visual impairment and this would agree with our findings where significant visual loss was found in patients with severe DMI grades. Macular perfusion may be only one of several factors affecting vision in DR. Early and more sensitive changes reflected in measures such as impaired colour vision, contrast sensitivity, and electroretinographic abnormalities have been demonstrated whilst visual acuity was preserved [19]. Early changes in retinal function have been reported in diabetic patients before retinal vascular changes can be detected and this suggests neurodegenerative events could precede vascular changes [20].

In terms of anatomical structural changes, photoreceptor layer integrity, and outer nuclear layer involvement with cystic spaces have been found to correlate better with visual acuity than macular thickness [21, 22]. We did not find a statistically significant correlation between visual acuity and CRT or the presence of intraretinal cysts. A Mann-Whitney $U$ test showed no difference in CRT between eyes with intraretinal cysts and those without cysts $(p=0.29)$ in our 
study. DRIL has been shown to correlate more consistently with visual acuity in active and resolved centre involving DMO [23]. We found a strong correlation between the presence of DRIL and visual acuity.

Visual acuity change over time has been associated with DRIL change over time, and strong correlations exist between baseline DRIL and subsequent visual acuity after macular oedema resolution [24]. Radwan et al. found the same correlations between DRIL and visual acuity did not exist for eyes with centre involved macular oedema from other conditions [24]. Resolution of post cataract surgery and uveitis associated macular oedema is often associated with functional improvement in vision [25]. Resolution of DMO may not always be associated with visual improvement and vision may paradoxically worsen [26].

Although histologic correlations of OCT derived DRIL have not been demonstrated, DRIL may represent disruptions of synaptic connections between bipolar, horizontal, and amacrine cells [27]. It has been postulated that vascular changes induced by diabetes in the inner retina may result in structural changes manifest as DRIL on OCT [9]. Pelosini et al. described a correlation between the volume of retinal tissue between the inner and outer plexiform layers and visual acuity in a series of patients with cystoid macular oedema including DMO [28]. Their results showed the volume of tissue up to $1000 \mu \mathrm{m}$ from the foveal centre predicted $80 \%$ of visual acuity whereas central macular thickness predicted only $14 \%$ of visual acuity [28]. A separate analysis of non DMO eyes was not performed. An association between visual acuity and IS/OS junction integrity in eyes with DMO is well established $[29,30]$. It is likely that both transduction elements represented by outer retinal structural damage as well as transmission elements represented by axonal integrity contribute to visual loss in DMO [31].

OCTA has been used to quantify changes in retinal microvasculature, and semiautomated algorithms have been used to calculate indices of microvascular density and morphology in segmented and non-segmented images [32].

Segmentation issues in the presence of DMO make standardised evaluation and quantification challenging. Various different commercial devices use different algorithms to compute OCTA images with various post-processing steps after analysis. Large scale comparative studies are required to determine whether quantifiable metrics applied to vasculature on OCTA are comparable between devices.

Segmentation artefacts are recognised as being a limiting factor in the interpretation of OCTA images. In addition, low flow may not be reliably detected and erroneously recorded as absence of flow. Reproducibility of measurements in studies may be variable. Bradley et al. showed a high degree of intergrader agreement for DMI grades when evaluating OCTA images in a small-scale retrospective study [33].
The limitations of our study were its cross-sectional retrospective design and small sample size; however, our patients largely did not have macular thickening which may confound some measures such as DRIL. We did not measure axial length to correct for individual retinal magnification and this may have affected the accuracy of FAZ measurements. Axial length measurement has been shown to influence OCTA derived measurements in a series of healthy eyes [34]. In the study by Sampson et al. a different OCTA device was utilised and the deep retinal plexus was not evaluated limiting comparison with our study [34]. We did exclude patients with refractive errors above +2.00 and -2.00 dioptres spherical equivalent, which may negate the effects of significant image magnification.

Integrating OCTA into longitudinal rather than cross sectional studies may be more useful to monitor individual disease progression or predict response to treatment. We did not include factors such as duration of disease, HbAlc measurements, and hypertension in the regression analysis which may have influenced results.

Prospective studies will be required to validate our results and to define in more detail the utility of OCTA in clinical practice.

\section{Summary}

\section{What was known before}

- Diabetic macular ischaemia is a feature of diabetic maculopathy, defined by enlargement of the foveal avascular zone and macular capillary non-perfusion and is associated with reduced visual acuity.

- Optical coherence tomography angiography is a noninvasive imaging technique which can distinguish retinal capillary layers and allow microvascular assessment.

\section{What this study adds}

- FAZ area, DMI grade, EZ disruption, and DRIL length correlate with visual acuity and could be incorporated into longitudinal clinical assessment of individual patients with diabetic retinopathy.

\section{Compliance with ethical standards}

Conflict of interest The authors declare that they have no conflict of interest.

Publisher's note Springer Nature remains neutral with regard to jurisdictional claims in published maps and institutional affiliations. 


\section{References}

1. Klein BE. Overview of epidemiologic studies of diabetic retinopathy. Ophthalmic Epidemiol. 2007;14:179-83.

2. Yau JW, Rogers SL, Kawasaki R, Lamoureux EL, Kowalski JW, Bek T, et al. Global prevalence and major risk factors of diabetic retinopathy. Diabetes Care. 2012;35:556-64.

3. Sim DA, Keane PA, Zarranz-Ventura J, et al. Predictive factors for the progression of diabetic macular ischaemia. Am J Ophthalmol. 2013;156:684-92.

4. Classification of diabetic retinopathy from fluorescein angiograms. ETDRS report number 11. Early treatment Diabetic Retinopathy Study Research Group. Ophthalmology. 1991;98:807-22.

5. Jonas JB, Schneider U, Naumann GO. Count and density of human retinal photoreceptors. Graefes Arch Clin Exp Ophthalmol. 1992;230:505-10.

6. Samara WA, Say EA, Khoo CT, et al. Correlation of foveal avascular zone size with foveal morphology in normal eyes using optical coherence tomography angiography. Retina. 2015;35:2188-95.

7. Magrath GN, Say EAT, Sioufi K, et al. Variability in foveal avascular zone and capillary density using optical coherence tomography angiography machines in healthy eyes. Retina. 2017;37:2102-11.

8. Laatikainen L, Larinkari J. Capillary-free area of the fovea with advancing age. Investig Ophthalmol Vis Sci. 1977;16:1154-7.

9. Balaratnasingam C, Inoue M, Ahn S, McCann J, Dhrami-Gavazi E, Yannuzzi LA, et al. Visual acuity is correlated with the area of the foveal avascular zone in diabetic retinopathy and retinal vein occlusion. Ophthalmology. 2016;123:2352-67.

10. Tan CS, Lim LW, Chow VS, Chay IW, Tan S, Cheong KX, et al. Optical coherence tomography angiography evaluation of the parafoveal vasculature and its relationship with ocular factors. Investig Ophthalmol. 2016;57:224-34.

11. Bresnick GH, Condit R, Syrjala S, Palta M, Groo A, Korth K. Abnormalities of the foveal avascular zone in diabetic retinopathy. Arch Ophthalmol. 1984;102:1286-93.

12. Samara WA, Shahlaee A, Adam MK, Khan MA, Chiang A, Maguire JI, et al. Quantification of diabetic macular ischaemia using optical coherence tomography angiography and its relationship with visual acuity. Ophthalmology. 2017;124:235-44.

13. Tam J, Dhamdhere KP, Tiruveedhula P, Manzanera S, Barez S, Bearse MA Jr, et al. Disruption of the retinal parafoveal capillary network in type 2 diabetes before the onset of diabetic retinopathy. Investig Ophthalmol. 2011;52:9257-66.

14. Krawitz BD, Mo S, Geyman LS, Agemy SA, Scripsema NK, Garcia PM, et al. Acircularity index and axis ratio of the foveal avascular zone in diabetic eyes and healthy controls measured by optical coherence tomography. Vis Res. 2017;139:177-86.

15. Dupas B, Minvielle W, Bonnin S, Couturier A, Erginay A, Massin $\mathrm{P}$, et al. Association between vessel density and visual acuity in patients with diabetic retinopathy and poorly controlled type 1 diabetes. JAMA Ophthalmol. 2018;136:721-8. https://doi.org/10. 1001/jamaophthalmol.2018.1319.

16. Salz DA, de Carlo TE, Adhi M, et al. Select features of diabetic retinopathy on swept source optical coherence tomographic angiography compared with fluorescein angiography and normal eyes. JAMA Ophthalmol. 2016;134:644-50.

17. Schottenhamml J, Moult EM, Ploner S, et al. An automatic, intercapillary area-based algorithm for quantifying diabetes related capillary dropout using optical coherence tomography angiography. Retina. 2016;36:593-S101.

18. Tang F, Sun Z, Wong R, Lok J, Lam A, Tham CC, et al. Relationship of intercapillary area with visual acuity in diabetes mellitus: an optical coherence tomography angiography study. $\mathrm{Br}$ J Ophthalmol. 2018. https://doi.org/10.1136/bjophthalmol-2018312010.

19. Tzekov R, Arden GB. The electroretinogram in diabetic retinopathy. Surv Ophthalmol. 1999;44:53-60.

20. Laron M, Bearse MA, Bronson-Castain K, et al. Interocular symmetry of abnormal multifocal electroretingrams in adolescents with diabetes and no retinopathy. Investig Ophthalmol Vis Sci. 2012;53:316-21.

21. Shin HJ, Lee SH, Chung H, Kim HC. Association between photoreceptor integrity and visual outcome in diabetic macular oedema. Graefes Arch Clin Exp Ophthalmol. 2012;250:61-70.

22. Ghazi NG, Scruggs RT, Batchelet AR, et al. Diabetic macular oedema. Ophthalmology. 2012;119:2643.e1.

23. Sun JK, Lin MM, Lammer J, et al. Disorganisation of the retinal inner layers as a predictor of visual acuity in eyes with centerinvolve diabetic macular oedema. JAMA Ophthalmol. 2014;132:1309-16.

24. Radwan SH, Soliman AZ, Tokarev J, Zhang L, van Kuijk FJ, Koozekanani DD. Association of disorganisation of retinal inner layers with vision after resolution of center-involved diabetic macular oedema. JAMA Ophthalmol. 2015;133:820-5.

25. Tranos PG, Tsaousis KT, Vakalis AN, Asteriades S, Pavesio CE. Long term follow up of inflammatory cystoid macular oedema. Retina. 2012;32:1624-8.

26. Browning DJ, Glassman AR, Aiello LP, et al. Diabetic Retinopathy Clinical Research Network. Relationship between optical coherence tomography- measured central retinal thickness and visual acuity in diabetic macular oedema. Ophthalmology. 2007; 114:525-36.

27. Sun JK, Radwan SH, Soliman AZ, Lammer J, Lin MM, Prager $\mathrm{SG}$, et al. Neuronal retinal disorganisation as a robust marker of visual acuity in current and resolved diabetic macular edema. Diabetes. 2015;64:2560-70.

28. Pelosini L, Hull CC, Boyce JF, McHugh D, Stanford MR, Marshall J. Optical coherence tomography may be used to predict visual acuity in patients with macular edema. Investig Ophthalmol Vis Sci. 2011;52:2741-8.

29. Maheshwary AS, Oster SF, Yuson RM, Cheng L, Mojana F, Freeman WR. The association between percent disruption of the photoreceptor inner segment-outer segment junction and visual acuity in diabetic macular edema. Am J Ophthalmol. 2010;150:63-7.

30. Otani T, Yamaguchi Y, Kishi S. Correlation between visual acuity and foveal microstructural changes in diabetic macular oedema. Retina. 2010;30:774-80.

31. Pelosini L, Hull CC, Boyce JF, McHugh D, Stanford MR, Marshall J. Author response: Predictors of visual acuity in Macular oedema. Investig Ophthalmol Vis Sci. 2012;53:924.

32. Kim AY, Chu Z, Shahidzadeh A, Wang RK, Puliafito CA, Kashani AH. Quantifying microvascular density and morphology in diabetic retinopathy using spectral-domain optical coherence tomography angiography. Investig Ophthalmol Vis Sci. 2016;57:362-70.

33. Bradley PD, Sim DA, Keane PA, Cardoso J, Agrawal R, Tufail A, et al. The Evaluation of diabetic macular ischaemia using optical coherence tomography angiography. Investig Ophthalmol Vis Sci. 2016;57:626-31.

34. Sampson DM, Gong P, An D, Menghini M, Hansen A, Mackey $\mathrm{DA}$, et al. Axial length variation impacts on superficial retinal vessel density and foveal avascular zone area measurements using optical coherence tomography angiography. Investig Ophthalmol Vis Sci. 2017;58:3065-72. 